\title{
Nephrectomy is necessary in the treatment of metastatic renal cell carcinoma
}

\author{
Tamer Abou Youssif, MD; Simon Tanguay, MD, FRCSC
}

\begin{abstract}
A Ithough we are observing a significant stage migration with an increasing number of patients being diagnosed with small renal masses confined to their kidney, roughly $20 \%$ of our patients have metastatic disease at the time of diagnosis. The therapeutic options available for this group of patients changed significantly during the last 5 years. The development and approval of new drugs for patients with disseminated disease forces us to re-evaluate the benefits associated with our previous therapeutic strategies. Although cytoreductive nephrectomy was largely accepted as a necessary step in the treatment of metastatic renal cell carcinoma (RCC), this concept is now challenged with the use of targeted therapy. Many believe we should abandon cytoreductive nephrectomy and adopt a pure systemic treatment approach. Before we endorse this concept, we must question the scientific rationale used in the past to justify performing cytoreductive nephrectomy and see if this rationale can be applied to targeted therapy.
\end{abstract}

\section{Clinical evidence to support cytoreductive nephrectomy}

The adoption of cytoreductive nephrectomy as a valid step in the treatment of metastatic RCC comes from 2 randomized phase III trials evaluating the role of cytoreductive nephrectomy. Both the Southwest Oncology Group (SWOG) 8949 and the European Organization for Research and Treatment of Cancer (EORTC) 30947 trials concluded that radical nephrectomy followed by interferon- $\alpha$ had an overall survival advantage over the use of interferon- $\alpha$ alone., ${ }^{1,2}$ The SWOG 8949 trial demonstrated a 3-month median survival advantage in favour of patients undergoing cytoreductive nephrectomy, whereas the EORTC 30947 concluded on an overall survival benefit of 10 months. Interestingly, when both trials were combined, nephrectomy did not improve the clinical response to interferon- $\alpha .^{3}$ The overall response rate were $6.9 \%$ and $5.7 \%$ for the nephrectomy plus interferon- $\alpha$ and interferon alone group, respectively $(p=0.6)$. Despite this poor response to interferon- $\alpha$, the overall survival of 13.6 months in patients treated with nephrectomy followed by interferon- $\alpha$ compared favorably to the 7.8 months observed in patients treated with inter- feron- $\alpha$ alone $(p=0.002)$. The survival benefit observed in these 2 trials constitutes strong evidence supporting the benefit of cytoreductive nephrectomy. Retrospective analysis of matched patients treated with interleukin-2 (IL-2) alone or IL-2 following a cytoreductive nephrectomy also support the role of surgical resection of primary tumours in this patient population. ${ }^{4}$ Most reports support that patients with good performance status are more likely to benefit from cytoreductive nephrectomy and confirmed an acceptable morbidity and mortality associated with surgery. Most patients, being able to initiate systemic immune therapy within an acceptable period of time, discredit the arguments suggesting that cytoreductive nephrectomy will significantly delay or prevent administration of systemic treatment.

In a recent population-based study using the Surveillance, Epidemiology and End Results (SEER) database, Zini and colleagues evaluated the impact of cytoreductive nephrectomy on survival of patients with metastatic RCC. ${ }^{5}$ Although this evaluation could not account for the potential impact of systemic therapy on survival, it supports the benefit of surgery in metastatic RCC. In their retrospective evaluation of over 5000 metastatic RCC patients treated with or without cytoreductive nephrectomy, matched and unmatched analysis confirmed a significant benefit in both cancer specific or overall survival. The 1- and 5-year cancer-specific survival for patients treated with nephrectomy were $53.6 \%$ and $19.4 \%$ compared with $18.5 \%$ and $2.3 \%$ in the nosurgery group. The 2.5 fold increased in cancer specific survival in this population-based analysis support the observed survival benefit in previous studies. This benefit was not related to performance status or increased co-morbidities.

\section{Immune modulation in RCC}

The close relationship between kidney cancer and the immune system has been an important research focus for many years. The rare but well-documented spontaneous regression of metastasis observed in less than $1 \%$ of patients following radical nephrectomy supported a special interaction between renal cancer and the host immune system.

Animal models of renal cancer support the hypothesis that tumour growth is associated with a progressive inhibition of 
the host immune system. Using the Renca model, Chagnon and colleagues demonstrated a progressive inhibition of $\mathrm{T}$ cell proliferation, interferon- $\gamma$ production and natural killer (NK) cell activity associated with tumour growth. ${ }^{6}$ These changes were associated with a progressive reduction in the ability to activate NF- $\kappa \mathrm{B}$ in splenic T cells. This immune suppression was postulated to be directly associated with tumour growth.

The immune suppression observed in animal models is also present in patients with RCC. Many reports demonstrated the ability of RCC to release immunosuppressive factors such as TGF- $\beta$, IL-10, gangliosides and prostaglandins. ${ }^{7,8}$ These factors contribute to the decrease in both dendritic (DC) and T cell functions observed in patients with RCC. Dendritic cells represent a key element in the generation of specific immune response to most pathogens including developing cancers. Gigante and colleagues confirmed the impact of developing RCC on this subset of essential immune modulators. ${ }^{9}$ They observed that patients with renal cancer have an important reduction in the number of circulating DCs compared to healthy individuals. In addition, DCs were found in large numbers within tumour tissue but displayed an immature phenotype and were unable to transform into mature DCs. The absence of maturation markers in tumour infiltrating antigen presenting cells support the inability of these cells to stimulate an appropriate immune response. Down stream to DC function are effector $\mathrm{T}$ cells. $\mathrm{Ng}$ and colleagues demonstrated an increased apoptosis and alteration in the NF- $\mathrm{KB}$ activation pathways in T cells of patients with RCC. ${ }^{8}$ The immune suppression mediated by soluble factors released by renal cancer is supported by the reversal of the immune changes observed after nephrectomy. The evidence supporting the presence of soluble factors released by renal cancers and the spontaneous improvement in immunologic parameters following radical nephrectomy constitute in part the scientific foundation to support the role of cytoreductive nephrectomy in patients with metastatic RCC. This evidence also supports the improved survival demonstrated in randomized phase III trials in the absence of improved response rate to systemic therapy. It is likely that debulking nephrectomy leads to a significant decrease in circulating immunosuppressive molecules and allows the immune system to mount a temporary response contributing to improved survival in some patients.

\section{Cytoreductive nephrectomy in the targeted therapy era}

Since the availability of a number of new molecules, such as tyrosine kinases and m-TOR inhibitors, the role of cytoreductive nephrectomy has been questioned. The occasional observed response in the primary tumour certainly contributes to reinforce the dilemma. Many physicians believe cytoreductive nephrectomy should at best be reserved only for patients responding to targeted therapy. It is true that our current level of evidence only supports the use of nephrectomy in patients where IFN- $\alpha$ therapy is planned. Currently IFN- $\alpha$ alone being no longer indicated or used and largely replaced by targeted therapy, should we repeat clinical trials in order to have level 1 evidence to support surgery in these patients. Using the RAND/UCLA appropriateness ratings, experts considered cytoreductive nephrectomy appropriate in patients with good surgical risks, tumourrelated symptoms and limited metastatic burden. ${ }^{10}$ These experts consider performing surgery in patients with poor surgical risk in the absence of symptoms to be inappropriate. All other scenarios were judged of uncertain benefit.

It is normal that our constant search for evidence-based approaches to support our recommendations make us question the role of cytoreductive nephrectomy. However, it remains clear for most cancers that multimodality approach is required to achieve cure. In light of the body of evidence supporting the impact of cytoreductive nephrectomy on the immune response and the fact that it most likely contributed to the improved survival observed in both the SWOG and EORTC trials, we should continue to consider nephrectomy as an integral part of our treatment plan. Cytoreductive nephrectomy should continue to be performed in selected patients with good performance status and limited metastatic burden. The observed response in primary tumours with targeted therapy supports the option of using systemic therapy first in patients where surgery is less likely to have an impact.

One should however keep in mind that targeted therapy alone does not offer cure to our patients and nephrectomy will always need to be performed in order to achieve cure.

From McGill University Health Centre, Montréal, QC

Competing interests: None declared.

This paper has been peer-reviewed.

\section{References}

1. Flanigan RC, Salmon SE, Blumenstein BA et al. Nephrectomy followed by interferon alfa-2b compared with interferon alfa-2b alone for metastatic renal-cell cancer. N Engl J Med 2001;345:1655-9.

2. Mickisch GH, Garin A, van Poppel H, et al. Radical nephrectomy plus interferon alfa-based immunotherapy compared with interferon alfa alone in metastatic renal-cell carcinoma: a randomized trial. Lancet 2001;358:966-70.

3. Flanigan RC, Mickisch G, Sylvester R, et al. Cytoreductive nephrectomy in patients with metastatic renal cancer: a combined analysis. J Urol 2004;171:1071-6.

4. Belldegrun A, Shvarts 0, Figlin RA. Expanding the indications for surgery and adjuvant interleukin-2-based immunotherapy in patients with advanced renal cell carcinoma. Cancer I Sci Am 2000;6Suppl1:S88-92. 
5. Zini L, Capitanio U, Perrotte P, et al. Population-based assessment of survival after cytoreductive nephrectomy versus no surgery in patients with metastatic renal cell carcinoma. Urology 2009;73:342-6.

6. Chagnon F, Tanguay S, Ozdal OL, et al. Potentiation of a dendritic cell vaccine for murine renal cell carcinoma by CpG oligonucleotides. Clin Cancer Res 2005; 11:1302-11.

7. Kudo A, Rayman P, Horton C, et al. Gangliosides expresses by renal cell carcinoma cell line SK-RC-45 are involved in tumour-induced apoptosis of T cells. Cancer Res 2003;63:1676-83.

8. $\mathrm{Ng}$ CS, Novick AC, Tannenbaum CS, et al. Mechanisms of immune evasion by renal cell carcinoma: tumour-induced T-lymphocyte apoptosis and NFkB suppression. Urology 2002;59:9-14.

9. Gigante $M$, Blasi A, Loverre $A$, et al. Dysfunctional DC subsets in RCC patients: Ex vivo correction to yield an effective anti-cancer vaccine. Mol Immunol 2008;46:893-901.
10. Halbert RJ, Figlin RA, Atkins MB, et al. Treatment of patients with metastatic renal cell cancer: a RAND Appropriateness Panel. Cancer 2006;107:2375-83.

Correspondence: Dr. Simon Tanguay, Department of Surgery (Urology), McGill University Health Centre, 1650 Cedar Ave., L8-318, Montréal, QC H3G 1A4; fax: 514-934-8297; simon.tanguay@mcgill.ca 